\title{
Work-related physical activity and psychological distress among women in different occupations: a cross-sectional study
}

\author{
Rhiannon Lee White ${ }^{1 *}$, Jason Bennie ${ }^{2}$, Gavin Abbott $^{3}$ and Megan Teychenne ${ }^{3}$
}

\begin{abstract}
Background: Recent evidence suggests that work-related physical activity may not have the same mental health benefits as leisure-time physical activity. Further, work-related physical activity is likely to include a variety of different behaviours for people with different occupations. As such, the aim of this study was to determine if occupation type moderated the association between work-related physical activity and psychological distress.

Methods: A randomly selected sample of 1080 women from Melbourne, Australia completed the International Physical Activity Questionnaire (IPAQ) and General Health Questionnaire (GHQ-30), and reported their current occupation.

Results: Linear regression analyses indicated that occupation significantly moderated the association between work-related walking and psychological distress $(F[8,55]=2.26, p=.036)$. Given evidence of moderation, we fitted linear regression models to test the associations between work-related physical activity and psychological distress for three separate groups; professionals, sales and services workers, and tradespersons. Female tradespersons who engaged in a low ( $B=-3.81, p=.006)$ or high amount of work-related walking ( $B=-3.23, p=.029)$, had significantly lower psychological distress symptoms than those who engaged in no work-related walking. There were no significant associations between work-related physical activity of any intensity and psychological distress for professionals, or sales and service workers.
\end{abstract}

Conclusions: Given the relationship does not exist across all occupations, work-related physical activity should not be promoted above and beyond leisure-time physical activity. However, walking at work may be important in reducing psychological distress for some people and should therefore, not be discounted.

Keywords: Exercise, Mental health, Occupations, Physical activity, Psychological distress, Work, Tradesperson

\footnotetext{
* Correspondence: Rhiannon.White@westernsydney.edu.au

'School of Health Sciences, Western Sydney University, Locked Bag 1797, Penrith, NSW 2751, Australia

Full list of author information is available at the end of the article
}

(c) The Author(s). 2020 Open Access This article is licensed under a Creative Commons Attribution 4.0 International License, which permits use, sharing, adaptation, distribution and reproduction in any medium or format, as long as you give appropriate credit to the original author(s) and the source, provide a link to the Creative Commons licence, and indicate if changes were made. The images or other third party material in this article are included in the article's Creative Commons licence, unless indicated otherwise in a credit line to the material. If material is not included in the article's Creative Commons licence and your intended use is not permitted by statutory regulation or exceeds the permitted use, you will need to obtain permission directly from the copyright holder. To view a copy of this licence, visit http://creativecommons.org/licenses/by/4.0/. The Creative Commons Public Domain Dedication waiver (http://creativecommons.org/publicdomain/zero/1.0/) applies to the data made available in this article, unless otherwise stated in a credit line to the data. 


\section{Background}

Mental health disorders contribute a substantial proportion to the global burden of disease, and account for $10.4 \%$ of the total all-cause disability adjusted life years and an even larger $18.9 \%$ of years lived with a disability [1]. Despite increased efforts to understand and prevent mental health disorders, and promote mental health, the gap in life expectancy between the general population and those with a mental health disorder continues to widen [2]. As such, the World Health Organization declared the need for collaborative public health approaches to reduce the pervasive and costly effects of mental health disorders [3]. Abundant evidence shows that physical activity is associated with a reduced risk of mental ill-health, including depression, anxiety, and psychological distress [4]. However, despite ample evidence supporting a relationship between physical activity and mental health, the strength of the relationship varies considerably across studies. This inconsistency needs to be understood so that physical activity can be optimally used to promote mental health and reduce mental illhealth.

Studies show that the relationship between physical activity and mental health varies between different physical activity domains (i.e., areas of life in which physical activity can occur), with meta-analytic evidence demonstrating an inverse association between leisure-time physical activity and mental ill-health only [5]. That meta-analysis also demonstrated that work-related physical activity may in fact be associated with an increased likelihood of depression, anxiety, and psychological distress [5]. However, large heterogeneity existed between the results of individual studies. Additionally, research now shows that higher amounts of work-related physical activity are associated with poor physical health and an increased risk of early mortality [6]. As such, workrelated physical activity might not have the same benefits to health and wellbeing as physical activity in other domains, particularly leisure-time.

Despite evidence suggesting that work-related physical activity may not be as beneficial as leisure-time physical activity, and may potentially be detrimental $[5,6]$, global [7] and national physical activity guidelines [8,9] encourage adults to be active during any life domain, including leisure-time physical activity, transportation, occupational physical activity, and household chores. Evidence suggests that leisure-time physical activity only accounts for a small portion of adults' weekly physical activity, with occupational physical activity being the largest contributor [10]. Also, adults with lower levels of education, who often have poorer mental health, engage in more work-related physical activity, compared to adults with higher levels of education [11]. Moreover, approximately half of all people who report no leisure- time physical activity, report 60 min or more of workrelated physical activity per day [12]. This suggests that many people may complete the recommended amount of physical activity per week without completing any leisure-time specific physical activity and therefore, may receive no mental health benefit. Further, a recent cohort study showed that compensation occurs when adults transitioned from a sedentary occupation to an active occupation, as participants reduced their physical activity during leisure-time to account for the increased activity at work [13]. While this compensation may result in the same amount of weekly physical activity, it may be associated with poorer mental health if the same mental health benefits are not derived from work-related physical activity, when compared to leisure-time physical activity. However, given the limited studies investigating the association between work-related physical activity and mental health, further evidence is needed. This is especially true for female populations given work-related physical activity has been more frequently examined among male populations. Further, evidence suggests that women report higher levels of psychological distress [14], and typically engage in lower levels of leisure-time physical activity [15] compared to men. Despite engaging in lower levels of leisure-time physical activity [15], data shows that $40 \%$ of males and $38 \%$ of females participate in strenuous work-related physical activity most or all of the time [11]. Given women's participation in paid employment has increased steadily since the 1960's [16, 17], and their employment is as likely to include physical activity, work-related physical activity needs to be better understood among women in terms of psychological distress and wellbeing.

While measuring domain-specific physical activity helps to distinguish different types of physical activity behaviour (e.g., work and leisure), and take context into account, work-related physical activity is still likely to include within it a large variety of different behaviours and movements. For example, an office worker who is mainly seated during desk-based work activities may engage in light walking throughout the day, while typical workrelated physical activity for those working in a construction site could include frequent bouts of low to moderate intensity movement, including walking, standing, and lifting. Not only is the behaviour itself likely to be physically different, but, the purpose of the activity and the context surrounding the behaviour is likely to vary also. As such, it seems plausible that the relationship between work-related physical activity and mental health may vary between different occupations. However, to our knowledge, no study has taken into account occupation type, when examining the relationship between workrelated physical activity and mental health related outcomes. Therefore, the purpose of this study was to 
examine the relationships between work-related physical activity and psychological distress among women, and determine if occupation type moderated these relationships.

\section{Methods}

\section{Participants}

Participants were recruited from 45 neighbourhoods (suburbs) in Melbourne, Australia as part of a larger study $[18,19]$. The 45 suburbs were selected based on the Australian Bureau of Statistics' Socioeconomic Index for Areas (SEIFA) which reflects the level of socioeconomic advantage and disadvantage of each suburb based on education, occupation, income, household status, and access to resources [20]. A total of 15 suburbs of low-SEIFA were randomly selected, 15 of mid-SEIFA, and 15 of high-SEIFA. Women living in those suburbs between 18 and 65 years were then randomly selected from the electoral roll and 4800 women were sent a questionnaire and invited to participate. The study was approved by the Deakin University Human Research Committee and a total of 1554 women returned the questionnaire and provided consent to participate (32.38\% response rate). We excluded 389 participants as they were full-time students or unemployed at the time of data collection, and a further 85 who did not report psychological distress, leaving 1080 participants. There were no significant differences between those who were excluded due to not responding to specific questionnaire items and those included in the analysis in terms of selfreported health $(t=-1.68, p=.09)$, BMI $(t=.72, p=.48)$, or socioeconomic advantage $(t=-.15, p=.88)$.

\section{Measures}

\section{Work-related physical activity}

Participants self-reported their work-related physical activity by completing the International Physical Activity Questionnaire - Long Form (IPAQ-L). The IPAQ-L involves seven-day recall of physical activity across four domains (leisure-time, work, transport, and household physical activity). Participants reported the number of days (frequency), and hours and minutes on each day (duration), in each physical activity domain across the past week. Using a standardised protocol [21], total duration of work-related physical activity was calculated by multiplying the frequency and duration within this domain only. This process was completed for each intensity of physical activity to calculate walking, moderate, and vigorous work-related physical activity variables. Total work-related physical activity was then calculated by adding all three intensities together. All four variables had a sizable proportion of zeroes and were consequently recoded into categorical variables based on tertile splits [22]. The IPAQ-L has been shown to have acceptable one-week test-retest reliability (Spearman's rho $=0.80$ ) and moderate criterion validity (median rho $=$ $0.30)$ using accelerometery as the standard [21].

\section{Psychological distress}

We used the 30-item version of the General Health Questionnaire (GHQ-30) to assess participants' level of psychological distress [23]. The GHQ-30 is a widely used questionnaire that has been extensively validated in adult populations [24] and asks participants if they recently (in the last couple of weeks) experienced a range of psychiatric symptoms. Participants respond to each symptom by selecting the appropriate option indicating better/ healthier than normal (scored 0), the same as usual (scored 0), worse/more than usual (scored 1), or much worse/more than usual (scored 1). The GHQ-30 total score ranging from 0 to 30 is commonly used as a continuous variable $[25,26]$ and has been highly correlated with the Present State Examination index [27]. Alternatively, a threshold total score of $>4$ indicates participants at risk of developing psychiatric disorders [28]. As such, we used the validated cut-off score to categorise participants as at-risk or not at-risk in descriptive analyses but, used the total score as a continuous variable for all regression analyses.

\section{Occupation category}

Based on classification similar to the International Standard Classification of Occupations (2008), participants reported their main occupation as manager or administrator, professional, associate professional, clerical, sales, or service worker, tradesperson, production or transport worker, or labourer. We grouped similar occupations together to create three occupational categories to test for moderation: 1) managers, administrators, and professionals, 2) clerical, sales, and service workers, and 3) tradespersons, transport workers, and labourers. For brevity, these three groups of participants will be referred to as Job Category 1: 'Professionals', Job Category 2: 'Sales and Service', and Job Category 3: 'Trades'.

\section{Potential confounders}

As sociodemographic and lifestyle factors are likely to influence physical activity [15] and psychological distress [29-31], we adjusted inferential analyses for age, education, self-reported height and weight-derived Body Mass Index (BMI), self-reported presence of a physical illness, injury, or disability; and leisure-time physical activity.

\section{Data analysis}

All data analyses were conducted in STATA 14 (StataCorp, TX). Descriptive statistics were used to report participant characteristics (mean, standard deviation, frequencies) for the entire sample, followed by 
descriptive statistics for each of the three subsamples (i.e., professionals, sales and service, and trades). To test overall associations between work-related physical activity and psychological distress, linear regression analyses were conducted using the total sample, where the GHQ30 total score (psychological distress) was the dependent variable and the work-related physical activity measures (in separate models) were independent variables. To examine whether the effect of work-related physical activity on psychological distress differed across occupation categories, we fitted our linear regression models with a main effect of occupation category and its interaction with work-related physical activity. Where there was evidence of a moderating effect of occupation category on the relationship between work-related physical activity on depressive symptoms $(p<.05$ for interaction effect), we conducted linear regression analyses stratified by occupation category to examine associations between work-related physical activity and psychological distress for professionals, sales and service, and trades separately. We fitted all regression models with cluster-robust standard errors to account for clustering within suburbs, and excluded participants with missing total (16.20\%), walking $(19.35 \%)$, moderate $(17.04 \%)$, or vigorous
(17.22\%) physical activity data in the regression model with that corresponding dependent variable.

\section{Results}

Participants $(n=1080)$ were 18 to 65 year old females $(M=41.24, \quad S D=11.69)$ and were mostly born in Australia (75.9\%). The majority of participants were married (66.2\%) and nearly half had a university degree (43.6\%). Just over half were within a healthy weight range $(57.0 \%)$, and nearly one-third (29.2\%) of participants were at risk of psychological distress (Table 1), which is similar to previous findings among Australian women [32]. Participants' self-rated health was also similar to results from a previous mail-out survey study conducted in Australia with a much higher $60 \%$ response rate [33]. Approximately one-third of participants resided in a neighbourhood of high-SEIFA (34.35\%), while slightly more were from a mid-SEIFA neighbourhood (39.38\%) and slightly less from a low-SEIFA neighbourhood (26.28\%). Participants with trade-related occupations reported more work-related physical activity per week $(M=20.17 \mathrm{~h})$ than professionals $(M=6.96 \mathrm{~h})$ or sales and service workers $(M=9.04 \mathrm{~h})$. Table 1 shows physical activity levels of participants across light,

Table 1 Physical activity levels and psychological distress across different job categories

\begin{tabular}{|c|c|c|c|c|}
\hline & $\begin{array}{l}\text { Total Sample }(N=1080) \\
N(\%)\end{array}$ & $\begin{array}{l}\text { Professionals }(N=597) \\
N(\%)\end{array}$ & $\begin{array}{l}\text { Sales and Service }(N=349) \\
N(\%)\end{array}$ & $\begin{array}{l}\text { Trades }(N=134) \\
N(\%)\end{array}$ \\
\hline \multicolumn{5}{|l|}{ Work-related physical activity } \\
\hline \multicolumn{5}{|l|}{ Total (hrs/week) } \\
\hline Low (<0.28 h p/week) & $302(32.4)$ & $194(36.5)$ & $101(34.2)$ & $7(6.7)$ \\
\hline Medium (0.28-7.33 h p/week) & $305(32.8)$ & $190(35.8)$ & $97(32.9)$ & $18(17.1)$ \\
\hline High (> 7.33 h p/week) & $324(34.8)$ & $147(27.7)$ & $97(32.9)$ & $80(76.2)$ \\
\hline \multicolumn{5}{|l|}{ Walking (hrs/week) } \\
\hline No walking & $461(41.1)$ & $238(43.5)$ & $129(41.9)$ & $28(26.2)$ \\
\hline Low (0.1-3.5 h p/week) & $235(29.1)$ & $166(30.3)$ & $91(29.5)$ & $23(21.5)$ \\
\hline High (> 3.5 h p/week) & $260(29.8)$ & $143(26.1)$ & $88(28.6)$ & $56(52.3)$ \\
\hline \multicolumn{5}{|l|}{ Moderate (hrs/week) } \\
\hline No moderate PA & $461(48.2)$ & $292(54.2)$ & $154(50.2)$ & $15(13.6)$ \\
\hline Low (0.1-2.67 h p/week) & $235(24.6)$ & $132(24.5)$ & $78(25.4)$ & $25(22.7)$ \\
\hline High (> 2.67 h p/week) & $260(27.2)$ & $115(21.3)$ & $75(24.4)$ & $70(63.6)$ \\
\hline \multicolumn{5}{|l|}{ Vigorous (hrs/week) } \\
\hline No vigorous PA & $623(64.3)$ & $389(70.9)$ & $196(63.4)$ & $38(34.2)$ \\
\hline Low (0.1-3 h p/week) & $178(18.4)$ & $101(18.4)$ & $60(19.4)$ & $17(15.3)$ \\
\hline High (> 3 h p/week) & $168(17.3)$ & $59(10.7)$ & $53(17.2)$ & $56(50.5)$ \\
\hline \multicolumn{5}{|l|}{ At risk of psychological distress } \\
\hline Yes $(>4)$ & $315(29.2)$ & $156(26.1)$ & 118 (33.8) & 41 (30.6) \\
\hline $\mathrm{No}(\leq 4)$ & 765 (70.8) & 441 (73.9) & $231(66.2)$ & $93(69.4)$ \\
\hline
\end{tabular}

PA physical activity 
Table 2 Associations between work-related physical activity and psychological distress

\begin{tabular}{|c|c|c|c|}
\hline & B & $95 \% \mathrm{Cl}$ & $p$ \\
\hline \multicolumn{4}{|l|}{ Total } \\
\hline Medium (0.28-7.33 h p/week) vs. Low (<0.28 h p/week) & 0.05 & $-0.67,0.77$ & .887 \\
\hline High (> $7.33 \mathrm{~h} \mathrm{p} /$ week) vs. Low (<0.28 h p/week) & 0.18 & $-0.53,0.88$ & .620 \\
\hline High (> $7.33 \mathrm{~h}$ p/week) vs. Medium (0.28-7.33 h p/week) & 0.12 & $-0.60,0.85$ & .732 \\
\hline \multicolumn{4}{|l|}{ Walking } \\
\hline Low walking (0.1-3.5 h p/week) vs. No walking & -0.20 & $-0.88,0.48$ & .561 \\
\hline High (> $3.5 \mathrm{~h}$ p/week) vs. No walking & -0.27 & $-1.00,0.47$ & .471 \\
\hline High (> 3.5 h p/week) vs. Low walking (0.1-3.5 h p/week) & -0.07 & $-0.79,0.65$ & .849 \\
\hline \multicolumn{4}{|l|}{ Moderate } \\
\hline Low PA (0.1-2.67 h p/week) vs. No moderate PA & 0.15 & $-0.83,1.12$ & .764 \\
\hline High PA (> 2.67 h p/week) vs. No moderate PA & -0.03 & $-0.81,0.76$ & .948 \\
\hline High PA (> 2.67 h p/week) vs. Low PA (0.1-2.67 h p/week) & -0.17 & $-1.25,0.90$ & .750 \\
\hline \multicolumn{4}{|l|}{ Vigorous } \\
\hline Low PA (0.1-3 h p/week) vs. No vigorous PA & 0.18 & $-0.79,1.15$ & .709 \\
\hline High PA (>3h p/week) vs. No vigorous PA & 0.20 & $-0.73,1.13$ & .667 \\
\hline High PA (> $3 \mathrm{~h}$ p/week) vs. Low PA (0.1-3 h p/week) & 0.02 & $-1.17,1.15$ & .973 \\
\hline
\end{tabular}

Separate models were tested for each work-related physical activity measure (i.e., walking, moderate, vigorous, total). For rows one and two in each model, the reference category is the lowest PA category (i.e., no PA or low PA). For row three, the highest category is compared to the middle PA category. All models were adjusted for neighbourhood clustering as well as for age, BMI, education status, physical illness, and leisure-time physical activity. PA physical activity

moderate, and vigorous categories, and full participant characteristics are included in Supplementary Material.

\section{Work-related physical activity and psychological distress}

No physical activity variables (walking, moderate, vigorous, or total) were significantly associated with psychological distress in the whole sample (Table 2). However, interaction analyses revealed that job category significantly moderated the association between work-related walking and psychological distress $(\mathrm{F}[8,55]=2.26$, $p=.036)$. Job category did not significantly moderate the association between psychological distress and moderate $(\mathrm{F}[8,55]=0.43, p=.898)$, vigorous $(\mathrm{F}[8,55]=0.68$, $p=.709)$, or total $(\mathrm{F}[8,55]=1.00, p=.447)$ work-related physical activity.

After adjustment for potential confounders, those in trade-related occupations engaging in a high volume (> $3.5 \mathrm{~h}$ per week) of walking at work had an estimated 3.23 points lower $(p=.029)$ psychological distress score compared to those doing no work-related walking (Table 3).

Table 3 Associations between work-related walking and psychological distress stratified by occupation category

\begin{tabular}{|c|c|c|c|}
\hline & B & $95 \% \mathrm{Cl}$ & $p$ \\
\hline \multicolumn{4}{|l|}{ Professionals } \\
\hline Low walking (0.1-3.5 h p/week) vs. No walking & 0.11 & $-0.65,0.87$ & .768 \\
\hline High (> $3.5 \mathrm{~h}$ p/week) vs. No walking & 0.03 & $-0.97,1.02$ & .954 \\
\hline High (> $3.5 \mathrm{~h}$ p/week) vs. Low walking (0.1-3.5 h p/week) & -0.08 & $-1.01,0.84$ & .857 \\
\hline \multicolumn{4}{|l|}{ Sales and Service } \\
\hline Low walking (0.1-3.5 h p/week) vs. No walking & 0.29 & $-1.20,1.79$ & 695 \\
\hline High (> $3.5 \mathrm{~h}$ p/week) vs. No walking & 0.53 & $-1.05,2.12$ & .500 \\
\hline High (> $3.5 \mathrm{~h}$ p/week) vs. Low walking (0.1-3.5 h p/week) & 0.24 & $-1.07,1.55$ & .713 \\
\hline \multicolumn{4}{|l|}{ Trades } \\
\hline Low walking (0.1-3.5 h p/week) vs. No walking & -3.81 & $-6.47,-1.14$ & .006 \\
\hline High (> $3.5 \mathrm{~h}$ p/week) vs. No walking & -3.23 & $-6.12,-0.34$ & .029 \\
\hline High (> $3.5 \mathrm{~h}$ p/week) vs. Low walking (0.1-3.5 h p/week) & 0.57 & $-2.40,3.55$ & .698 \\
\hline
\end{tabular}

Separate models were tested for each work-related physical activity measure (i.e., walking, moderate, vigorous, total). For rows one and two in each model, the reference category is the lowest PA category (i.e., no PA or low PA). For row three, the highest category is compared to the middle PA category. All models were adjusted for neighbourhood clustering as well as for age, BMI, education status, physical illness, and leisure-time physical activity 
Similarly, those engaging in a lower amount $(0.1-3.5 \mathrm{~h}$ per week) of walking at work had an estimated 3.81 points lower $(p=.006)$ psychological distress score compared to those doing no work-related walking. There were no significant associations between work-related walking and psychological distress for professionals or sales and service workers.

\section{Discussion}

This is the first study to compare different occupations in terms of the relationship between work-related physical activity and psychological distress. The key finding was that among a large sample of women, those with trade, labour, or transport-related occupations, who reported some work-related walking, have significantly lower psychological distress than those who reported no work-related walking. However, in contrast, there were no significant associations between work-related physical activity of any intensity and psychological distress for 'professionals' (i.e., professionals, admin workers, or managers); or 'sales and service workers' (i.e., clerical, sales, and services workers).

The finding that work-related walking was inversely associated with psychological distress among women working in trade occupations only, could potentially be explained by a sense of satisfaction. As job satisfaction is highly influenced by accomplishments at work [34], it is possible that achieving physical tasks at work provides a sense of accomplishment and satisfaction, which if experienced on a regular basis, is likely to lead to a higher sense of life satisfaction and reduced psychological distress. Among women this may be particularly true as material achievements are more strongly associated with job satisfaction than financial achievements [35]. A recent qualitative study examined people's perspectives on community gardening, and identified that participants experienced a sense of satisfaction because they contributed to something [36]. Participants also acknowledged that they experienced a great sense of satisfaction by being able to make a difference through their work [36]. While community gardening is a different setting to work, the notion of being satisfied by helping others and contributing to a larger goal that involves interaction and collaboration to build a project, may be similar for people with trade-related occupations whose work involves landscaping or construction, where the work completed leads to benefits for other people. This sense of satisfaction may also evoke similar emotions to experiencing a sense of mastery, which is hypothesised as a mechanism for the mental health benefits of physical activity, as a sense of mastery may benefit mental health due to enhanced feelings of control and success, and increased self-esteem [37]. While accomplishments, a sense of mastery, and job satisfaction are possible across all occupation types, and certainly all employees may experience varying levels of job satisfaction, it is possible that for women with trade-related occupations, the amount of physical activity at work is more closely related, or more integral to, job satisfaction, given these types of jobs are typically more active than other occupations. Among professional or sale related occupations, employees may experience a sense of satisfaction, but physical activity may not be as integral to employee accomplishments.

It is also possible that physical activity within traderelated occupations is more likely to occur outdoors than physical activity within professional or sale and service roles, meaning work-related physical activity for people in job category 3 may be more likely to involve exposure to greenspace. Time spent in greenspace has been associated with lower levels of depression, anxiety, and stress [38, 39] and physical activity outdoors has been associated with greater reductions in depression and anxiety, compared to physical activity indoors [40]. However, we did not assess exposure to greenspace, hence future research should examine whether exposure to greenspace moderates the association between work-related physical activity and mental health related outcomes.

Despite work-related physical activity being associated with reduced psychological distress, this was only the case for walking (i.e., light-intensity physical activity). This finding could be the result of a female only sample, as previous research [41] identified an inverse relationship between light-intensity physical activity and depression in women but an inverse relationship between vigorous-intensity physical activity and depression in men. However, it could also be that moderate and vigorous physical activity at work do not hold the same association with psychological distress as walking. Vigorous physical activity is sometimes associated with feelings of displeasure, particularly among people whose competence or confidence is lacking [42]. Further, the demand-control model [43] proposes that higher occupational demands, either psychological or physical, increase job stress and is inversely associated with psychological wellbeing [44]. Tasks at work that are more physically vigorous may be perceived as more demanding and may therefore lead to more adverse psychological outcomes, when compared to less vigorous tasks. Job control, or autonomy, however, may partially buffer the detrimental effects of job demands on psychological wellbeing [44]. It could be possible that different occupations provide employees with varying levels of job control and a lack of control enables job demands to lead to adverse psychological outcomes within some occupations more than others.

In relation to intensity, previous research also shows that affect was most positive among participants 
exercising at either lower intensities, or at a self-selected intensity [45]. The option to self-select the intensity of physical activity may not be as possible within the work domain, as it is during leisure-time - where moderate and vigorous physical activity are associated with better psychological outcomes $[5,46]$. Specifically within the work domain, the intensity of physical activity may not be a personal choice, and vigorous activities may undermine an employee's need for autonomy. Autonomy, which is "the need to self-regulate one's experiences and actions" [47], is associated with better mental wellbeing across all life domains, and autonomy support specifically within the workplace is associated with better psychological outcomes $[48,49]$. It may be possible that trade-related employees perceive greater autonomy around their involvement in physically active behaviours at work, or perhaps even greater autonomy or control over the intensity at which they complete physically active tasks. Further, autonomy satisfaction is associated with more autonomous motivation towards physical activity and autonomous motivation is consistently associated with psychological wellbeing [48]. Perhaps because physical activity is more integral to trade-related occupations, employees with these types of jobs are more autonomously motivated towards work-related physical activity. In other occupation types, employees may certainly be autonomously motivated towards their job in general, but physical activity at work is seen as more separable to the job itself or their reason for choosing such a job. However, no study has assessed autonomy support, or motivation specifically towards work-related physical activity. Perhaps future research should examine whether these factors moderate the association between work-related physical activity and mental health related outcomes.

While it remains unclear the reasons why work-related physical activity may hold different relationships with psychological distress among different occupations, the current study does suggest that physical activity at work may be differently associated with mental health depending on occupation type. While caution needs to be exercised due to the low response rate and the smaller group of participants working in trade-related occupations, in the future, physical activity guidelines may need to distinguish between leisure-time physical activity and workrelated physical activity $[5,6,50]$, as it appears that work-related physical activity may be not associated with mental health in the same consistent way as leisure-time physical activity $[5,6]$. Also, future research needs to examine why work-related physical activity is beneficial within some occupations and not others.

This study is the first to compare different occupations in terms of the relationship between work-related physical activity and psychological distress, and the first to show that occupation type moderates the association between work-related physical activity and psychological distress. This finding prompts a number of new research questions; nevertheless, a number of limitations should be noted. First, while the results are likely to be generalisable across different ages, since only female participants were included, these results may not be generalisable to males. Therefore, future research should examine the role of occupation type among men. Further, the sample in this study was overrepresented by women with a university degree and by women with professional occupations, when compared to populationbased data in Melbourne, Australia [51]. However, because of the three occupational categories, this bias is unlikely to impact the inverse association between workrelated physical activity and psychological distress for women with trade-related occupations. We do acknowledge that the findings for professionals and sales and service workers may have been different if the sample was more representative in terms education and occupation type. Nevertheless, the results still present novel findings and suggest future areas of inquiry. Also important to note is that the participants self-reported physical activity which may have introduced social desirability bias, and that there were less participants in occupation category 3 (i.e., tradespersons, transport workers, and labourers) compared to the other two occupation categories. While occupations were categorised with other occupations that were most similar, it is possible that the relationship between physical activity and mental health varies even between similar jobs. In the future, more specific occupation categories should be used. Lastly, a cross-sectional design means that causal relationships could not be determined.

\section{Conclusion}

Physical activity at work does not appear to be consistently associated with psychological distress as the relationship seems to vary between different occupations. As such, a number of mechanisms and potential moderators need to be investigated before we can truly understand how to best promote mental health through workplace physical activity. Nevertheless, walking at work for females with trade, labour, and transport-related occupations may reduce psychological distress symptoms, and therefore, work-related physical activity should not be completely disregarded in terms of its potential for improving mental health.

\section{Supplementary information}

Supplementary information accompanies this paper at https://doi.org/10. 1186/s12889-020-09112-7.

Additional file 1. Table 1. Participant Characteristics 


\section{Abbreviations}

IPAQ: International Physical Activity Questionnaire; GHQ-30: General Health Questionnaire; SEIFA: Australian Bureau of Statistics' Socioeconomic Index for Areas; BMI: Body Mass Index; PA: Physical activity

\section{Acknowledgements}

Not applicable.

\section{Authors' contributions}

RW led the conceptualisation of the study, undertook data analysis, and led the writing of the manuscript. JB was involved in the methodological design and writing of the manuscript. GA was involved in data analysis and writing of the manuscript. MT was involved in the conceptualisation of the study and writing of the manuscript. The authors read and approved the final manuscript.

\section{Funding}

The study on which these data are based was funded by the National Heart Foundation of Australia (Ref G02M 0658). However, the National Heart Foundation played no part in the design or conduct of the study; collection, management, analysis, or interpretation of the data; or preparation, review, or approval of the manuscript.

\section{Availability of data and materials}

The datasets generated and/or analysed during the current study are not publicly available due to ethical constraints.

\section{Ethics approval and consent to participate}

Ethics approval was received from the Deakin University Human Research Ethics Committee (EC 26-2002) and written consent was obtained from each person participating in the study.

\section{Consent for publication}

Not Applicable.

\section{Competing interests}

The authors declare that they have no competing interests.

\section{Author details}

${ }^{1}$ School of Health Sciences, Western Sydney University, Locked Bag 1797, Penrith, NSW 2751, Australia. ²Physically Active Lifestyles Research Group (USQ-PALs), Institute for Resilient Regions, University of Southern Queensland, Springfield, Central QLD 4300, Australia. ${ }^{3}$ Institute for Physical Activity and Nutrition (IPAN), School of Exercise and Nutrition Sciences, Deakin University, Geelong, Australia.

\section{Received: 30 March 2020 Accepted: 12 June 2020}

\section{Published online: 26 June 2020}

\section{References}

1. Whiteford HA, Ferrari AJ, Degenhardt L, Feigin V, Vos T. Global burden of mental, neurological, and substance use disorders: an analysis from the global burden of disease study 2010. In: JPatel V, Chisolm D, Dua T, Laxminarayan R, Medina-Mora ME, editors. Mental, neurological, and substance use disorders. 3rd ed. Washington: World Bank Group; 2015. p. $29-40$.

2. Lawrence D, Hancock KJ, Kisely S. The gap in life expectancy from preventable physical illness in psychiatric patients in Western Australia: retrospective analysis of population based registers. BMJ. 2013;346.

3. World Health Organization. The world health report: 2001: mental health: new understanding, new Hope. Geneva, Switzerland: World Health Organization:; 2001.

4. Rebar AL, Stanton R, Geard D, Short C, Duncan MJ. Vandelanotte C. A metameta-analysis of the effect of physical activity on depression and anxiety in non-clinical adult populations. Health Psychol Rev. 2015;9(3):366-78.

5. White RL, Babic MJ, Parker PD, Lubans DR, Astell-Burt T, Lonsdale C. Domain-specific physical activity and mental health: a meta-analysis. Am J Prev Med. 2017;52(5):653-66.

6. Coenen P, Huysmans MA, Holtermann A, Krause N, van Mechelen W, Strake LM, et al. Do highly physically active workers die early? A systematic review with meta-analysis of data from 193696 participants. Br J Sports Med. 2018; 52:1320-6.

7. World Health Organization. Global recommendations on physical activity for health. Geneva, Switzerland: WHO Press; 2010

8. Canadian Society for Exercise Physiology. Physical activity tips for adults (18-64 years). Ontario: Public Health Agency of Canada; 2011.

9. Department of Health and Ageing. Australia's physical activity and Sedendary behaviour guidelines. Canberra: Department of Health and Ageing; 2014

10. Jacobi D, Charles MA, Tafflet M, Lommez A, Borys JM, Oppert JM. Relationships of self-reported physical activity domains with accelerometry recordings in French adults. Eur J Epidemiol. 2009;24(4):171-9.

11. He XZ, Baker DW. Differences in leisure-time, household, and work-related physical activity by race, ethnicity, and education. J Gen Intern Med. 2005; 20(3):259-66.

12. Centers for Disease Control Prevention. Prevalence of leisure-time and occupational physical activity among employed adults--United States, 1990. Morbidity and Mortality Weekly Report. 2000;49(19):420.

13. Nooijen CF, Pozo-Cruz D, Nyberg G, Sanders T, Galanti MR, Forsell Y. Are changes in occupational physical activity level compensated by changes in exercise behavior? Eur J Public Health. 2018;28(5).

14. Slade T, Grove R, Burgess P. Kessler psychological distress scale: normative data from the 2007 Australian National Survey of mental health and wellbeing. Aust N Z J Psychiatry. 2011;45(4):308-16.

15. Bauman AE, Reis RS, Sallis JF, Wells JC, Loos RJ, Martin BW, et al. Correlates of physical activity: why are some people physically active and others not? 2012;380(9838):258-71

16. Crompton R, Brockmann M, Lyonette C. Attitudes, women's employment and the domestic division of labour: a cross-national analysis in two waves. Work Employ Soc. 2005;19(2):213-33.

17. Korpi W, Ferrarini T, Englund S. Women's opportunities under different family policy constellations: gender, class, and inequality tradeoffs in western countries re-examined. Soc Politics. 2013;20(1):1-40.

18. Ball K, Timperio A, Salmon J, Giles-Corti B, Roberts R, Crawford D. Personal, social and environmental determinants of educational inequalities in walking: a multilevel study. J Epidemiol Community Health. 2007;61(2):108-14.

19. Ball K, Timperio A, Crawford D. Neighbourhood socioeconomic inequalities in food access and affordability. Health Place. 2009;15(2):578-85.

20. Pink B. Socio-economic indexes for areas (SEIFA): technical paper. Australian Bureau of Statistics: Canberra; 2011.

21. Craig CL, Marshall AL, Sjöström M, Bauman AE, Booth ML, Ainsworth BE, et al. International physical activity questionnaire: 12-country reliability and validity. Medicine \& science in sports \& exercise. 2003;35(8):1381-95.

22. Teychenne M, Ball K, Salmon J. Associations between physical activity and depressive symptoms in women. Int J Behav Nutr Phys Act. 2008;5(27).

23. Jackson CJ. The general health questionnaire. Occup Med. 2007;57(1):79.

24. Shek DT. Factor structure of the chinese version of the general health questionnaire (GHQ-30): a confirmatory factor analysis. J Clin Psychol. 1993; 49(5):678-84.

25. Puustinen PJ, Koponen $H$, Kautiainen $H$, Mäntyselkä $P$, Vanhala $M$. Psychological distress measured by the GHQ-12 and mortality: a prospective population-based study. Scandinavian journal of public health. 2011;39(6): 577-81.

26. Steinmo S, Hagger-Johnson G, L. Bidirectional association between mental health and physical activity in older adults: Whitehall II prospective cohort study. Prev Med. 2014;66:74-9.

27. Banks MH. Validation of the General Health Questionnaire in a young community sample. Psychol Med. 1983;13(2):349-53.

28. Goldberg D, Williams P. A user's guide to the general health questionnaire. Windsor: NFER-Nelson; 1988.

29. Jeon GS, Jang SN, Rhee SJ, Kawachi I, Cho SI. Gender differences in correlates of mental health among elderly Koreans. The Journals of Gerontology Series B: Psychological Sciences Social Sciences. 2007;62(5):S323-S9.

30. Lincoln KD, Taylor RJ, Watkins DC, Chatters LM. Correlates of psychological distress and major depressive disorder among African American men. Res Soc Work Pract. 2011;21(3):278-88.

31. Rakovac M, Pedisic Z, Pranic S, Greblo Z, Hodak D. Sociodemographic and lifestyle correlates of health-related quality of life in Croatian university students. Appl Res Qual Life. 2013;8(4):493-509.

32. Department of Human Services. The Victorian population health survey. Victoria: Department of Human Services; 2004 
33. Kavanagh AM, Bentley R, Turrell G, Broom DH, Subramanian S. Does gender modify associations between self rated health and the social and economic characteristics of local environments? J Epidemiol Community Health. 2006; 60(6):490-5.

34. Griffin ML, Hogan NL, Lambert EG, Tucker-Gail KA, Baker DN. Job involvement, job stress, job satisfaction, and organizational commitment and the burnout of correctional staff. Crim Justice Behav. 2010;37(2):239-55.

35. Punnett BJ, Duffy JA, Fox S, Gregory A, Lituchy T, Miller J, et al. Career success and satisfaction: a comparative study in nine countries. Women Manag Rev. 2007;22(5):371-90.

36. Quested E, Thøgersen-Ntoumani C, Uren H, Hardcastle SJ, Ryan RM. Community gardening: basic psychological needs as mechanisms to enhance individual and community well-being. Ecopsychology. 2018;10(3): 173-80.

37. Paluska SA, Schwenk TL. Physical activity and mental health. Sports Med. 2000;29(3):167-80.

38. Beyer K, Kaltenbach A, Szabo A, Bogar S, Nieto F, Malecki Kljoer, et al. Exposure to neighborhood green space and mental health: evidence from the survey of the health of Wisconsin. Int J Environ Res Public Health. 2014; 11(3):3453-72

39. Cohen-Cline H, Turkheimer E, Duncan GE. Access to green space, physical activity and mental health: a twin study. J Epidemiol Community Health. 2015;69(6):523-9

40. Thompson Coon J, Boddy K, Stein K, Whear R, Barton J, Depledge MH. Does participating in physical activity in outdoor natural environments have a greater effect on physical and mental wellbeing than physical activity indoors? A systematic review. Environ Sci Technol. 2011;45:1761-72.

41. Lindwall M, Rennemark M, Halling A, Berglund J, Hassmén P. Depression and exercise in elderly men and women: findings from the Swedish national study on aging and care. J Aging Phys Act. 2007;15(1):41-55.

42. Biddle S, Batterham AM. High-intensity interval exercise training for public health: a big HIT or shall we HIT it on the head? Int I Behav Nutr Phys Act. 2015;12(1):95.

43. Karasek RA Jr. Job demands, job decision latitude, and mental strain implications for job redesign. Adm Sci Q. 1979;24(2):285-308.

44. Van der Doef M, Maes S. The job demand-control (-support) model and psychological well-being: a review of 20 years of empirical research. Work Stress. 1999;13(2):87-114.

45. Rose EA, Parfitt G. A quantitative analysis and qualitative explanation of the individual differences in affective responses to prescribed and self-selected exercise intensities. J Sport Exerc Psychol. 2007;29(3):281.

46. Teychenne M, Ball K, Salmon J. Physical activity and likelihood of depression in adults: a review. Prev Med. 2008;46(5):397-411.

47. Ryan RM, Deci EL. Self-determination theory: basic psychological needs in motivation, development, and wellness. New York: Guilford Publications; 2017.

48. Deci EL, Ryan RM. Facilitating optimal motivation and psychological wellbeing across life's domains. Can Psychol. 2008:49(1):14-34.

49. De Leersnyder J, Kim H, Mesquita B. Feeling right is feeling good: psychological well-being and emotional fit with culture in autonomy-versus relatedness-promoting situations. Front Psychol. 2015;6:630.

50. Teychenne M, White RL, Richards J, Schuch FB, Rosenbaum S, Bennie JA. Do we need physical activity guidelines for mental health: what does the evidence tell us? Ment Health Phys Act. 2020;18.

51. Australian Bureau of Statistics. Census of Population and Housing: General community profile: Greater Melbourne, Catalogue number 2001.0. Belconnen: Australian Bureau of Statistics; 2011.

\section{Publisher's Note}

Springer Nature remains neutral with regard to jurisdictional claims in published maps and institutional affiliations.

Ready to submit your research? Choose BMC and benefit from:

- fast, convenient online submission

- thorough peer review by experienced researchers in your field

- rapid publication on acceptance

- support for research data, including large and complex data types

- gold Open Access which fosters wider collaboration and increased citations

- maximum visibility for your research: over $100 \mathrm{M}$ website views per year

At BMC, research is always in progress.

Learn more biomedcentral.com/submissions 